\title{
Process selection methodology for near net shape manufacturing
}

\author{
Daniele Marini $^{1}$ - Jonathan R. Corney ${ }^{1}$
}

Received: 16 May 2019 / Accepted: 6 October 2019 / Published online: 14 December 2019

(C) The Author(s) 2019

\begin{abstract}
This paper presents a new selection methodology that for the first time supports the identification of Near Net Shape (NNS) processes. The methodology, known as "Product, Geometry, Manufacturing and Materials Matching" (ProGeMa3), is composed of four steps, which aim to minimize raw material usage and machining by adopting a NNS approach. A key component of the methodology is the Process Selection Matrix (ProSMa) that associates a component's shape and production volume with its material requirements to reduce the number of candidate NNS processes. A final selection is then made from this shortlist by using fuzzy logic and considering other constraints and functional requirements. The ProGeMa3 selection process is illustrated by its application to an industrial component that resulted in changes to the processes used for its commercial manufacture. The ProGeMa3 and ProSMa presented in this paper aspires to be current and comprehensive for solid metallic components produced by casting, forging and additive technologies. However, ProSMa is also accessible as an open source resource available for other researcher to extend and adapt.
\end{abstract}

Keywords Process selection · Selection matrix · Fuzzy logic $\cdot$ Near net shape manufacturing $\cdot$ Forging · Casting · Additive layer manufacturing

\section{Introduction}

In the last 30 years, the concept of manufacturability has been applied to many different processes in numerous industries. This has resulted in the emergence of several different "Design for Manufacturing" methodologies which have in common the aim of reducing production costs through the application of general manufacturing rules. Near net shape technologies have expanded these concepts, targeting mainly primary shaping process, such as casting or forging.

As new manufacturing methods emerge and established ones develop, the production engineer must constantly review the processes they employ to ensure they are the most appropriate. Ideally, an automated system would review the specifications of components and identify their optimum manufacturing process but in practice the shear range of options and the complex mix of interacting constrains (i.e. both quantitative and qualitative) make such automation difficult. So although various methodologies to support

Daniele Marini

daniele.marini@strath.ac.uk; daniele.marini19@gmail.com

1 Department of Design, Manufacturing and Engineering Management (DMEM), University of Strathclyde, 75 Montrose St, Glasgow G1 1XJ, UK manufacturing process selection have been reported, they have not been widely applied because of the resources and computational challenges required to implement them.

In response to these issues, this paper introduces a novel process selection methodology that is designed to review a company's portfolio of components and identify opportunities for adopting near net shape processes. Crucially, the approach described quickly focuses the review and on a small number of candidate parts and processes which are determined to be most economically and technically feasible. This initial filtering reduces the size of the search space to a point where computationally intensive methods can be effectively used to identify optimum solutions.

Near net shape (NNS) concepts are based on the work of [20, 37, 49, 72] and [3], who based their investigations mainly on metal casting and forging.

Near net shape is a relative property, rather than absolute, that defines a combination of product geometry (to be produced), material and manufacturing process (primary shaping process) to be minimal in raw material utilization and finishing machining operations, in comparison with other possible combinations [47].

However, the selection of an appropriate NNS process is often an "ad hoc" procedure focused on the manufacture of new components and based on the personal experience of the production engineers. In contrast, this paper introduces 
a systematic methodology for the selection of NNS process appropriate to an existing product.

Unlike previously reported approaches, this selection methodology uses product geometry, production volume and materials to classify candidate NNS processes. To make thisz feasible, the scope of the classification described is limited to:

- Metals (material constrain)

- Solid geometries (bars, tubes and prisms)

- Casting, forging and additive technologies (excluding joining and machining processes)

- Non-variable product requirements

Material wastage reduction and energy saving are evaluated by applying a NNS approach to manufacturing. However, other environmental factors, such as resource saving considerations (energy and water requirements), re-manufacturing (material recycling, reuse and repair) and waste management (e.g. hazardous wastage), related to manufacturing processes, are not considered in the developed process selection methodology. These factors can be quantified only subsequent to the feasibility stage, using supply chain management tools and product Life-Cycle Assessment (LCA). The links between circular economy and process selection have not yet been explored in literature. The quantitative evaluation of such factors is therefore beyond the scope of this paper. Similarly, the effect of product requirement modification on process selection has not been considered.

The following section reviews the main approaches reported for the selection of manufacturing processes based on material, production volume, component shape and technological and characteristics and other requirements comparable with process attributes.

Subsequently the Product, Geometry, Manufacturing, and Material Matching (ProGeMa3) Methodology is described and then applied to an industrial case study.

\subsection{Process and material selection in literature}

The generic process selection procedure usually has three steps: screening, ranking and a search for supporting information $[6,27]$. Seven general approaches have been identified for selection of the best process for a given material, design characteristics and product requirements:

1. Analytical

2. Probabilistic (Fuzzy Logic)

3. Knowledge Base System

4. Manufacturing and Product complexity

5. Methodological (Qualitative)

6. Optimization Algorithms

7. Topological (Numerical)

Considering each of these in turn, the analytical papers develop a multi-variable system of equations, quantifying the different process features and their capabilities of matching with the component requirements. This formulation allows the process with the resultant lowest cost to be considered as the best candidate.

For example, [2] develop a model based on manufacturing cost prediction. The model provides the material (i.e. considering only the product volume and the material cost) and basic processing costs, depending on the selected processes and cost is calculated through the cost time and production volume, using empirical constant. This cost refers to the production of an ideal component design for the selected process and a coefficient (relative cost coefficient) corrects the process cost considering the geometry to produce. The coefficient is composed of four parameters (determined though empirical graphs), associated with geometrical shape, section reduction/thickness, tolerances and surface finish giving the distance between the current and the ideal conditions. Swift and Booker [70] use the [2] formula, introducing a matrix for a preliminary screen of the processes. Other authors use cost functions and cost estimation for pre-selection screening: for example, [40] provide a measurement of casting process compatibility for the required production volume, weight input, thick/thin sections, tolerances, and surface finish. A simple proportion between the available capabilities and the requirements give a compatibility score. Every characteristic is weighted, depending on its importance, with a qualitative system. The casting processes are thus ranked depending on their compatibility values. Rao and Padmanabhan [58] use graph theory and matrix approach for screening the additive layer manufacturing processes. The combination of these two methods is able to deliver a multicriteria decision, defining the interactions between selection attributes.

The process attributes can be either qualitative or quantitative and their responses to the product requirements as well as their interrelation are summarized in an single index. For ranking the casting processes, [27] use the cost function with compatibility ranges for identifying the possible feasible processes and ranking them. From these first step, the best process is selected and, in a subsequent step, the process technological (tolerances, workable dimensions, surface roughness) and economical capabilities are matched, giving a complete overview of the process ability for produced the required product. The work is based on a previous investigation by [26], comparing target design and product requirements (material, size, product shape, mechanical precision and cost) with those that lie within the capacity of a large number of processes, seeking the subset which is capable of making the component. The subset is then ranked by economic criteria.

The probabilistic approach aims to develop a statistical correlation between the process capabilities and product 
requirements. In particular, fuzzy logic (artificial intelligence technology in control systems and pattern recognition) had been used by several authors. It is based on the observation that people make decisions based on imprecise and numerical information [19]. Fuzzy models, or sets, are mathematical means of representing vagueness and imprecise information, hence the term fuzzy [39]. Different from traditional probability, fuzzy sets are capable of representing, using and manipulating data that has a range of values, due to their uncertainness. Hence, in fuzzy logic, distinction between from full compatibility (one) and incompatibility (zero) is gradual between extreme ranges of the fuzzy set. Figure 1 illustrates the fuzzy logic approach. Several authors applied slightly different versions of fuzzy approach to process selection and decision making in manufacturing [19, $30,54,60,73]$. Where, $\left.L_{(\min }-a b s\right)$ is the absolute minimum value, $L_{m} i \mathrm{n}$ is the minimum typical, $L_{m} a x$ is the typical maximum value, and $\left.L_{(} \max -a b s\right)$ is the absolute maximum value of the investigated process's feature. $L_{r} e q$ is the requested value of product feature (e.g. required surface roughness). Compatibility assessment can be performed by mapping from qualitative description ('low', 'low to medium', 'medium', 'medium to high' and 'high') to numerical values.

Giachetti [30], Ravi [59] and Daws et al. [19] define compatibility by the requested value and four values which define the fuzzy set. If the requested value is outside of the set (4), compatibility is considered null. If it is in normal range, then the request is fully compatible (1). If value falls between normal and extreme ranges, then the value is intermediate between 0 and 1 , defined by a linear behaviour $(2,3)$.

$$
\begin{aligned}
& P_{L_{r e q}}=1, \text { if } L_{\text {min }}<L_{r e q}<L_{\text {max }} \\
& P_{L_{r e q}}=\frac{L_{r e q}--L_{\text {min }-a b s}}{L_{\text {min }}--L_{\text {min }-a b s}} \text { if } L_{\text {min }-a b s}<L_{r e q}<L_{\text {min }} \\
& P_{L_{r e q}}=\frac{L_{\text {max }-a b s}--L_{r e q}}{L_{a x-a b s} m-L_{\text {max }}}, \text { if } L_{\text {max }}<L_{r e q}<L_{\text {max }}-a b s \\
& P_{L_{r e q}}=0, \text { if } L_{r e q}<L_{\text {min-abs }}, \text { or } L_{r e q}>L_{\text {max }-a b s}
\end{aligned}
$$



Fig. 1 Fuzzy set for process capabilities (adapted from [59])
Using [22] possibility theory, [30] defines two different cases that occur in compatibility evaluation: possibility and necessity are defined. Possibility assesses to what extent a feature satisfies the request (optimistic selection strategy); on the other hand, necessity expresses to what extent a features certainly satisfies the query. The latter adapts pessimistic selection strategy by measuring the impossibility of the opposite event, determined using the complementary probability of the event itself.

Figure 2 illustrates the process of performing the calculations, using Eqs. 1 to 4 to determine the possibility and necessity values for a linear request. In order to evaluate values of possibility and necessity, a unique compatibility number is required, [30] used a factor called $\beta$ that represents the level of optimisms or pessimism that is acceptable to the decision maker. Factor $\beta$ is 1 for an optimist decision maker and 0 for a negative one (so always a value in the interval $\beta \in(0,1)$ ). A weighted average is calculated for each requirement between possibility and necessity values, mediated by factor $\beta$ (possibility) and $1-\beta$ (necessity). Using this methodology, a compatibility measure has been assigned to every process/product selection features. A geometric weighted mean is used for aggregating all $n$th compatibility values (5). Weight $(w)$ is assigned to every feature using linguistic values. Each of them is calculated as in Eq. 6.

$$
\begin{aligned}
& \left.\left.\left.P L_{(} r e q_{1}\right), L_{(} r e q_{2}\right),,, L_{\left(r e q_{n}\right.}\right)=\prod_{i=1}^{N} P\left(L_{r} e q_{i}\right)^{\left(r^{i}\right)} \\
& r=\frac{w_{i}}{\sum_{i=1}^{n} w_{i}}
\end{aligned}
$$

Giachetti [30] applies this theory to the first stages of product design and process selection, including a broad range of processes and material as possible candidates. Perzyk and Meftah [54] use fuzzy logic for developing design for manufacturability of a single component. Functional requirements, manufacturing rules and material processability are evaluated for a single component through a process index, taking into consideration evaluating production volume, appearance, surface properties, dimensional tolerances and material structure. The index is a triplet-type fuzzy number, which is combined with the ideal process (depending on the product requirements). Daws et al. [19] limit the search to casting processes, including investment, mould (permanent, ceramic and full), shell, sand, die and squeeze casting. Similarly, [60] apply a fuzzy logic approach to the cutting process selection that considers the material-thickness relation, cutting speed, piece complexity and process tolerance capabilities.

Knowledge-based systems use empirical data (usually collected in databases) in order to support selection process. Knowledge-based systems are usually flexible and leave 
Fig. 2 Schematic of possibility (left) and necessity (right) calculations for a linear requirement [30]

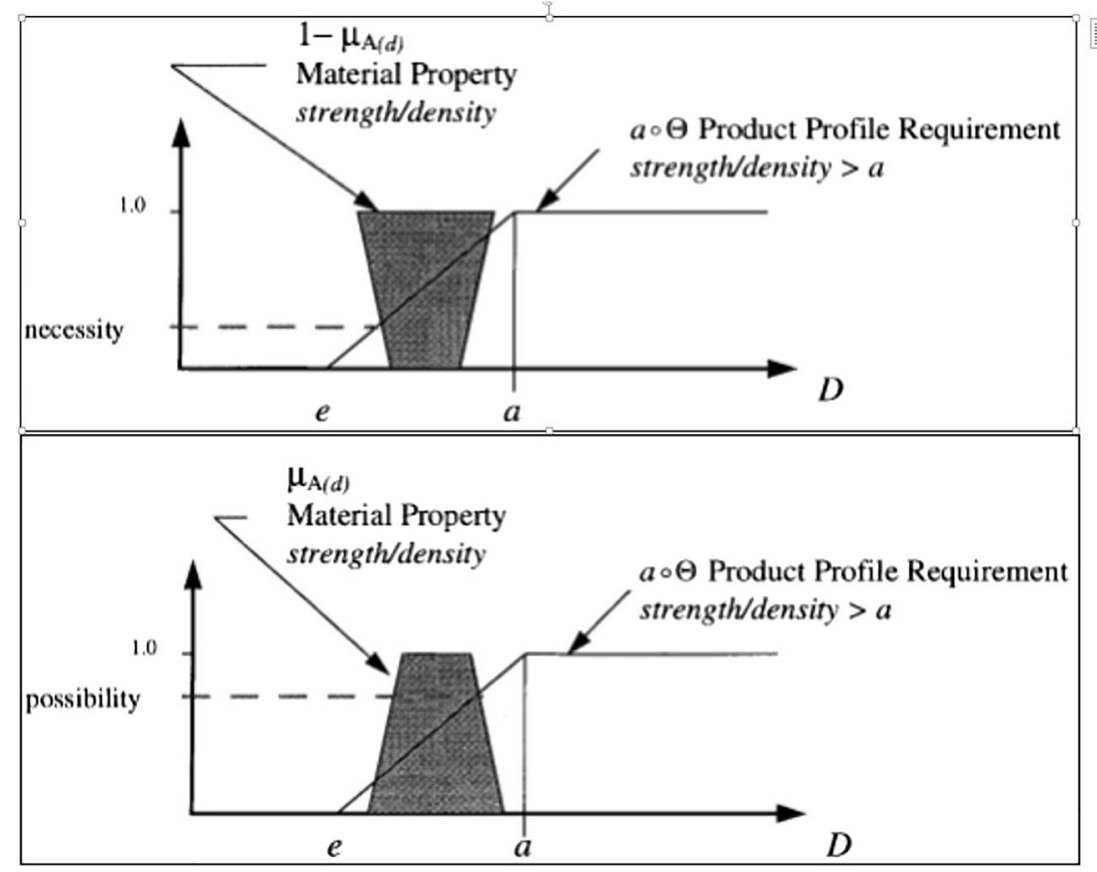

the decision making process to the user, providing all the required information to operate. A pioneering knowledgebased system in this area was reported by [65] to aid designers in choosing the best alloy and casting process for a particular set of specifications. The database displays both numerical and linguistic description of the processes, suitable for a certain material. The database includes a list of available material, selecting priorly to the manufacturing process (i.e. first list of processes are the material compatible ones). The designer selects qualitatively the best processes from its description, having excluded the unsuitable ones (i.e. relating to material and product specifications). Yu et al. [79] develop a computer-based routine which connects the geometrical factors, material and production factors attributes for identifying the most suitable process (i.e. selecting from casting, hot and cold forging processes). The algorithm uses a developed design compatibility analysis which quantifies the compatibility of every analysed category, by comparing the required values with datasets, for every considered process. Darwish and El-Tamimi [17] propose a knowledge-based algorithm for casting process selection, basing their decision criteria on design, production and manufacturing attributes. The author compare the process manufacturing attributes quantitatively (minimum thickness, tolerances, mass range, surface roughness, economic lot size), qualitatively (porosity, dimensional accuracy, mechanical properties) as well as the cost (tooling, labour, finishing and scrap costs). The available range of materials is used as a screening criteria for the processes. Similarly, [25] develop a system for casting process selection including a comparative cost routine
(Fig. 3). According to the previous papers, the authors screen the processes on different levels (casting alloy, geometric complexity, casting accuracy, production quantity and comparative costs). In contrast to other researchers, geometric complexity has been quantified through questions regarding the product (e.g. undercuts or internal holes presence). The selected material has been used as screening factor, taking into consideration the resulting and required mechanical properties. Xu et al. [78] develop a knowledgebased system for additive layer manufacturing, including the process cost as a decision criterion.

Complexity measurement is another tool adopted by researcher for quantifying and ranking manufacturing process in order to select the most suitable. The complexity approach logic defines the lowest process chain complexity (including process and product design) as easiest from manufacturing the component. Product complexity influences directly the manufacturing complexity, so an effective understanding of complexity nature and its relative measure can directly connect them. Product complexity increases with the number and diversity of "features" to be manufactured, as well as the nature and difficulty of the tasks required to produce the features [24]. Cooper et al. [16] have measured product complexity as a volume weighted average; meanwhile, [32] has used entropy for the information number evaluation. ElMaraghy and Urbanic [24] developed a complete formula for evaluating the product complexity by measuring its entropy (given by the information number and uniqueness o features) and the complexity of each of its features. Features and specification are defined and evaluated for every characteristic, assigning them a factor $(0$ 
Fig. 3 Casting process selection parametres and their interactions [25]

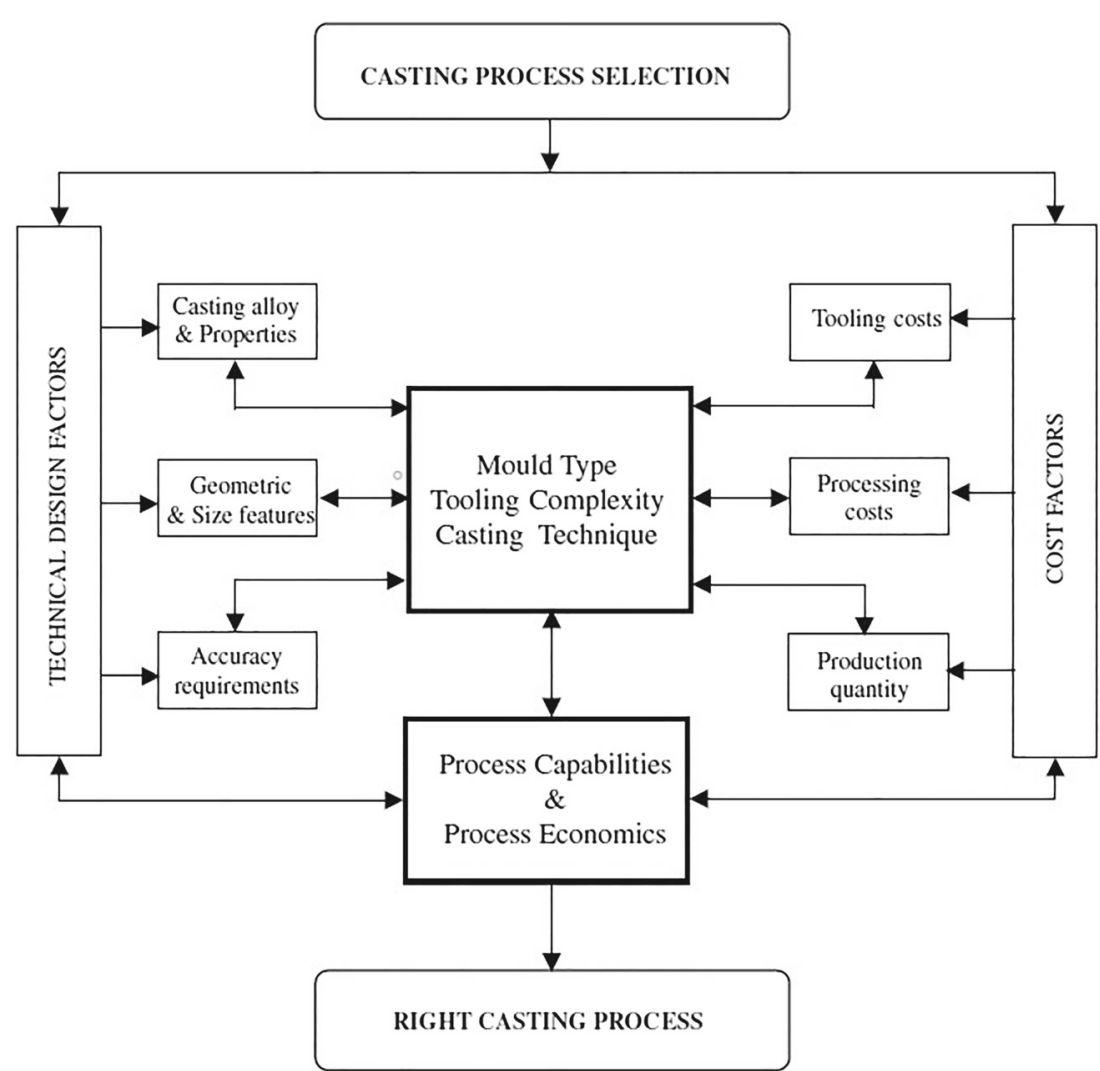

low effort, 0.5 medium effort, 1 high effort). All the factors are incorporated in the feature complexity coefficient and weighted by their percentage of presence in the component.

A matrix methodology is used to determine the relative complexity coefficient [24]. Complexity matrix describes all product characteristics and specifications. A factor indicates the relative effort to produce each of them or to perform the related task. Features and specification are defined and evaluated for every characteristic, assigning them a factor $(0$ low effort, 0.5 medium effort, 1 high effort). All the factors are incorporated in the feature complexity coefficient and weighted by their percentage of presence in the component.

The complexity index (obtained through the correspondent matrix) represents the difficulty of producing the component. A complexity index number does not have any meaning by itself. Comparing processes' complex indexes defines the closest one to the final shape, in terms of less needed manufacturing effort. Wiendahl and Scholtissek [75] expand the complexity concept to the whole manufacturing process, including product design, operation (process equipment, tools and labour) and structure. Similar to the previous authors, [48] quantity the manufacturing complexity using an entropic approach. Their model evaluates both the various component types and technologies used in a manufacturing system on the system's structural complexity. The authors apply their model by selecting the lowest complex manufacturing system configuration of an engine cylinder head. Kerbrat et al. [41] use the manufacturing complexity for evaluating how to combine subtracting and additive layer process for producing a mould. A modular CAD tool has been developed for comparing every single feature of the mould, selecting the less complex one to produce. Guenov [32] identifies two measuring systems for high-level decision makers. The aim is to compare alternatives during pre-competitive studies or during the architectural design process of composite systems. Similarly, the previous authors, the first measure is a complexity estimation of the Boltzmann's entropy, meanwhile the second measure is intended to estimate the costs and benefits related to system's performance.

Methodological investigations use a qualitative approach to determine the best process selection. The outputs of these papers consist usually in framework or flowcharts. For example, [1] develop a complex framework for material and process selection, taking into consideration the whole product life-cycle. The framework analyses the product life-cycle, diving it into three main phases: manufacturing, service and design/development. A dedicated part of the framework tries to rationalise the activities of requirements definition (design) and satisfaction (process). Xu et al. [77] develop a system for estimating the impact of different applications of rapid prototyping processes. Using product 
requirements and process cost, the methodology is able to quantify the process characteristics and compare different processes. Shercliff and Lovatt [64] define the interaction of process, material and design is peculiar to every category of processes (e.g. differences between casting and welding of an aluminium alloy). For the authors, the product requirements need to be matched one-byone with the process attributes: these requirements can be design-related (e.g. mechanical properties or dimensional characteristics), production-related (e.g. production volume and production rate) or processing-related. Similarly, the attributes can be process-, material- or design-related. A pair matching is evaluated on technical feasibility, avoiding in-process defects, product performance (i.e. final product characteristics) and economic bases. Different from all the other authors, [44] try to develop a connection between process modelling and process selection. They define the cost models and technical models mostly used in process selection, in order to validate the process candidate. Chakraborty and Dey [13] use the Quality Function Development (QFD) chart, usually called house of quality, for matching the technical and design requirements as well as connecting them with the customer requirements. The authors developed a total score from this well-known quality enhancement instrument, using a score matrix.

Some authors have been able to implement process selection into optimization algorithms developing complex models for assessing the process applicability. Working on reconfigurable manufacturing systems, [9] use genetic algorithms and a simulation-based optimization for process planning for a single product type, taking into consideration market demand fluctuation and minimum production volume (i.e. for making the production feasible). The functions to optimize have been defined as machine usage and change costs, configuration change cost, tool usage and change costs. A genetic algorithm (i.e. optimizing product design and machines data) and demand simulation software (i.e. providing) provide the most economic chain configuration. Vinodh et al. [74] apply a fuzzy analytic network, using different criteria for evaluating the best process and the best supplier to select. Qualitative scores have been assigned to different criteria for evaluating the process/supplier. A matrix assigns the value to the process for every criterion and the algorithm rank the different possible combination. The selected criteria are coefficients that belong to business improvement, product quality, supplier service and risk.

Topological models describe how elements (Finite Element Analysis) are bounded and connected. These numerical analyses are used for describing numerically the product features (e.g. using rules of proximity, the FEM elements identify an undercut). In this way, algorithm can assess all features of a component and assess the best process for realizing them. Holland et al. [36] develop a CAD-based algorithm that can identify cost-effective manufacturing options for metal forming. The matching is based on database shapes and features. The optimal and economic processes to associate with every feature are stored in database as well. The orientation of the feature is determined by the algorithm. This determines the most suitable process, defining the forming direction and the realizable features. A similar approach has been used by [43], developing a process-oriented forming features in cold extrusion to develop a process selection module (CAPP). The module is able to detect feature shape, main dimensions, and volumes, connecting them with the best suitable cold extrusion process option (i.e. giving also an indication of the stage numbers).

Material selection investigations can be taxonomized using the same categories of the process selection. Regarding process selection, the most part of the author use the workable material as screening for the available processes. However, some of the authors include material selection in their process selection method: [1] include a material selection in its methodology approach. Giachetti [30] use fuzzy logic also for material selection, using a variable request for predicting the different final properties. This allows the author to extend their probabilistic approach to the material selection. Brechet et al. [11] review the material selection methodology, pointing out the efficacy of the multi-objective criteria selection. Ashby has pioneered this field with several works (some of them extended to material selection). Ashby [6] identify firstly some material performance index for materials. The author develop instruments for material selection, mapping the Young modulus on the density of different materials and the linear expansion on the thermal conductivity. The mapping is dependent on the final product requirements (thermal distortion): dedicated procedures need to be developed in order to measure the material attributes for the particular product design. Ashby [5] applies single and multi-criteria optimization to material selection. The authors derive, from the objective function, some differential equations, using multiple input variables and boundary conditions as constraints. In the multi-criteria selection, the solution of the equations are trade-off Pareto surfaces. As single target, the author use the minimization of the component mass. In their applications, the author uses the multi-criteria for minimizing mass and cost (determining a Pareto trade-off solution) or using combined parameters to minimize (depending on the component requirements and functionality). For example, the author uses product of cost and density on the elastic limit (i.e. square root) and the density on the elastic limit (i.e. square root), forming another trade-off Pareto selection. Kutz [42] review some quantitative methods for material selection, pointing out the fundamental role of expert 
systems and numerical assistance (databases and knowledge base selection). Lately, the usage of stochastic and heuristic algorithms to material selection has rapidly increased. For example, [50] apply Analytic Network Process (ANP) to multi-criteria selection: the material characteristics taken under consideration are density, thermal attributes (operating temperature, conductivity), physical properties, fatigue and mechanical characteristic. The network is able to establish a ranking of different materials for single product requirements.

\subsection{Review synthesis}

In conclusion, fuzzy logic is capable of ranking the candidate processes in order of their features' compatibility with requested ones. Usually, these features include technological and other quantifiable requirements (e.g. tolerances, surface roughness), although it can be easily extended to every required feature (e.g. material usage, labour cost). The compatibility values are able to rank the processes and materials for the given requirements. Fuzzy logic is also able to quantify the compatibility qualitative features compatibility and deal with uncertainty.

Complexity approaches have similar potential, although their application appears to be oriented to product redesign and supply chain simplification. Similarly, topological optimization merges $\mathrm{CAD}$ and features identification, being currently used in many software packages. However, it fails to analyse complex problems, where uncertainty is present. Analytical models are less subjective and achieved highest precision in quantification of process/material compatibly, particularly when few features are considered. However, they are limited in dealing with uncertainty and complex connections between options. Further, analytical papers are limited to consider few selection criteria into their selections. Optimization papers overcome this problem by relying on probabilistic and analytical models, merging them with numerical capabilities and iteration.

Qualitative, methodological and knowledge-based approaches are most flexible and capable of dealing with complex interactions between material, design nd manufacturing process. However, the inability of quantifying feature's compatibility and generally low levels of subjectivity limits them to the selection of relatively restricted categories of process and materials.

\section{Product, Geometry, Manufacturing, and Material Matching (ProGeMa3) methodology}

The authors propose a NNS selection methodology that extends the capabilities of the reported systems (known as
Product, Geometry, Manufacturing, and Material Matching (ProGeMa3)). The methodology is illustrated schematically in Fig. 4. The aim of the methodology is to combine an existent product design with a combination of material and process in order to identify the most appropriate NNS manufacturing operation.

The methodology is composed of four main steps:

1. Economic opportunities screening: identifies opportunities for NNS applications

2. Material Selection: selects the material in relationship to its functional requirements

3. Process Screening Matrix (ProSMa): acting as a filter, sets viable processes for the combination of shape, material and production volume.

4. Process Compatibility Evaluation (Fuzzy logic): after the "static" selection tool (ProSMa), fuzzy logic acts with a "dynamic" selection the viable processes selected in the previous Step

Each of these steps are now described in detail:

Economic opportunities screening (step 1) is mainly devoted to screening and identifying components whose manufacturing costs could potentially be improved by application of alternative NNS processes. Each step of the component's manufacturing chain needs to be examined with aim of identifying production processes with the following features:

- High machining rate

- High raw material cost impact

- High production volume

- High lead time

The high complexity of the product design and manufacturing chain could be other factors in the identification of NNS opportunities. However, quantifying process chain complexity is difficult and consequently approximate evaluations have to be made in order to identify possible existing products to target. After this phase, the required information for the component production needs to be obtained.

Material selection (step 2) has been done subsequent to the components selected in step 1, using the method proposed by [6]. By using fuzzy logic, it is possible to select an optimal material based on the component requirements and usage conditions. In choosing this order of operations (i.e. material prior to process selection) ProGeMa3, similarly $[17,25,70]$, effectively limits the resulting number of combinations and interactions.

Process screening matrix (ProSMA) (step 3) examines the technical feasibility of candidate processes to reduce the number of possible manufacturing processes to investigate. Central to this step, a selection matrix (ProSMa), whose rows and columns are associated with input are the component's geometry and production volume selected in 
Fig. 4 Product Geometry, Manufacturing and Material Matching (ProGeMa3) Methodology schematization

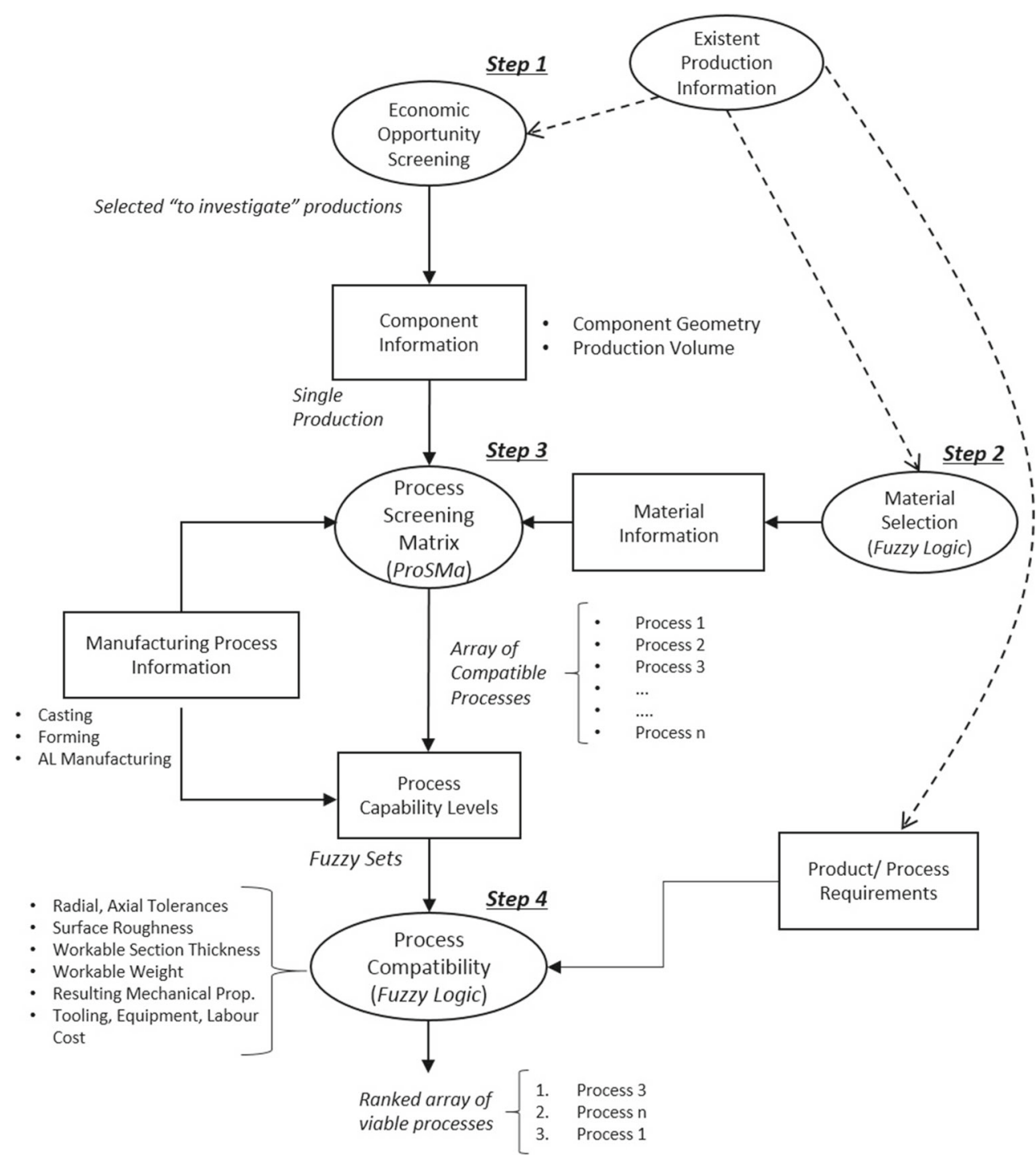

step 1 and the material, selected in step 2 (or, in alternative, the material currently in use), gives in output a set (array) of viable processes (which could include the processes used in the original component production).

Production volume, material and shape are classified in categories as follows:

- Material: irons, steel (carbon); steel (alloy, tool); stainless steel; copper and alloys; aluminium and alloys; magnesium and alloys; zinc and alloys; tin and alloys; lead and alloys; nickel and alloys; titanium and alloys.

- Production volume: very low (1 to 100$)$; low (100 to $1,000)$; low to medium (1,000 to 10,000$)$; medium to high $(10,000$ to 100,000$)$; high $(100,000+)$; all quantities.

- Component shape: 12 different component shapes can be selected, as showed in Table 1. The categories include three general geometric form (i.e. round, bar, tube) and five possible shapes derived from them (i.e. uniform cross section, change at the end, change at the centre, transverse element, and irregular).

The material and production volume categories are adapted from [39, 61] and [70].

The identification of the shape from the Table 1 is a qualitative assessment of existent shape in comparison with the general cases.

ProSMa is presented in Tables 2, 3, 4 and 5. The matrix is an extension of the [70] work, although their PRIMA matrix uses as input only the production volume and material. The number of casting and forming processes defined in [70] matrix do not take into consideration innovative technologies, whereas ProSMa include process such as semi-solid metal casting processes. Also is ProSMA, the additive layer manufacturing processes have been added. The ProSMa construction is based on the process review from $[7,8,10,18,21,23,28,29,31,33-35,38,51-53,55$, $56,61-63,66,69,70,76,80]$. 
Table 1 Component shape selected combinations and nomenclature, adapted from [61]

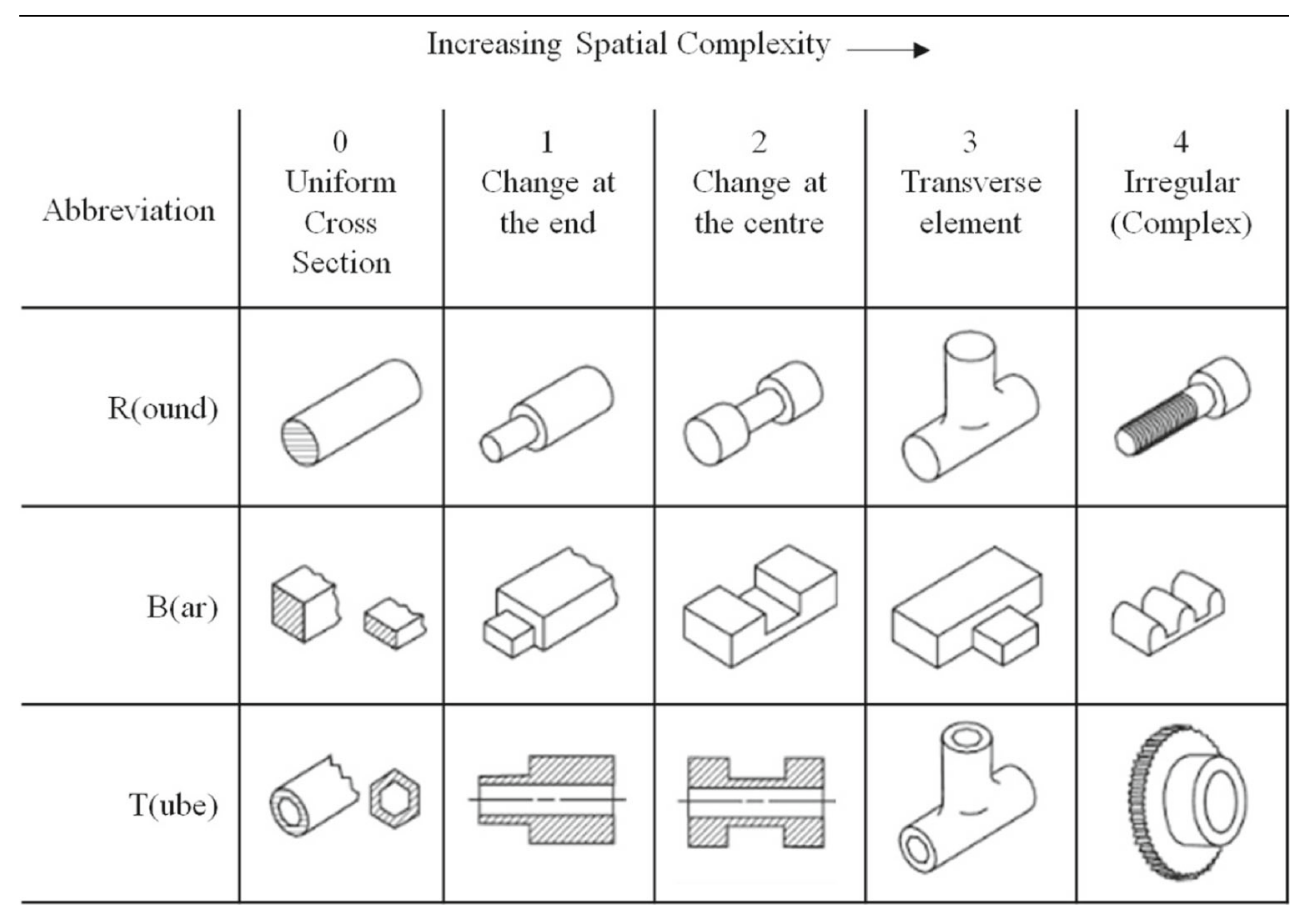

The manufacturing processes in output have been indexed and divided in three macro-categories as follows:

- Casting: sand casting(C.1); shell moulding (C.2); plaster moulding (C.3); lost foam casting (C.4); investment casting (C.5); ceramic mould casting (C.6); gravity-die casting (C.7); gravity-die casting (C.8); vacuum-die casting (C.9); pressure die casting (C.10); true-centrifugal casting (C.11); semi-centrifugal casting (C.12); centrifuge casting (C.13); squeeze casting (C.14); thixocasting, rheocasting (C.15); thixoforming (C.16).

- Forming: open-die forging (F.1); closed-die forging (F.2); isothermal forging (F.3); precision forging (F.4); cold forming (F.5); injection forging (F.6); rotary forging (F.7); shear forming (F.8); flow forming (F.9); hydroforming (F.10); powder forging (F.11); isostatic pressing (F.12); metal injection moulding (F.13).

- Additive Layer Manufacturing: selective laser sintering (SLS) (AM.1); selective laser melting (SLM) (AM.2); direct metal laser sintering (DMLS) (AM.3); electron beam melting (EBM) (AM.4); laser-based metal deposition (LBDM) (AM.5); electron beam-based metal deposition (EBMD) (AM.6); plasma deposition manufacturing (PDM) (AM.7).

The ProSMa is intended to be used for existing products that are designed as a single component part. Referring to Table 1, for all Round (R), Barr (B) and Tubular (T) basic geometries, the irregular (complex) shape (classified as 4) is meant to absorb all the cases that are not included in the other categories (uniform cross section, change at the end, change at the centre, transverse element). If the shape cannot be identified from its spatial complexity (not associable to any of the categories form 0 to 4 ), all the process for the identified basic geometry (Round, Barr or Tube) should be taken into consideration (all from 0 to 4 ) for the considered material and production volume.

The ProSMa can be used as guidance for mapping the manufacturing implications of design changes (passing from a geometric category to a different one). While ProSMa is not meant to be a tool for generating new product designs (given the difficulty of representing all the possible functional features) and similarly cannot be used for joining components or assembly processes. However, it can provide guidance for the manufacturing of merged geometries (e.g. passing from two distinct simple components to a single one).

Process compatibility evaluation (stage 4) uses fuzzy logic to enable identification of the most suitable manufacturing processes from the viable ones, selected in stage 3 . This stage has a dual function:

- Final screening: the processes that form particular features of components are excluded at this stage (e.g. thickness section).

- Process ranking: all the viable processes are ranked in order of their compatibility (between product requirements and process capabilities).

The fuzzy logic approach allows these two objectives to be achieved by associating the request with a four level fuzzy description of the process capabilities. The process capabilities are described by four levels and trapezoidal 


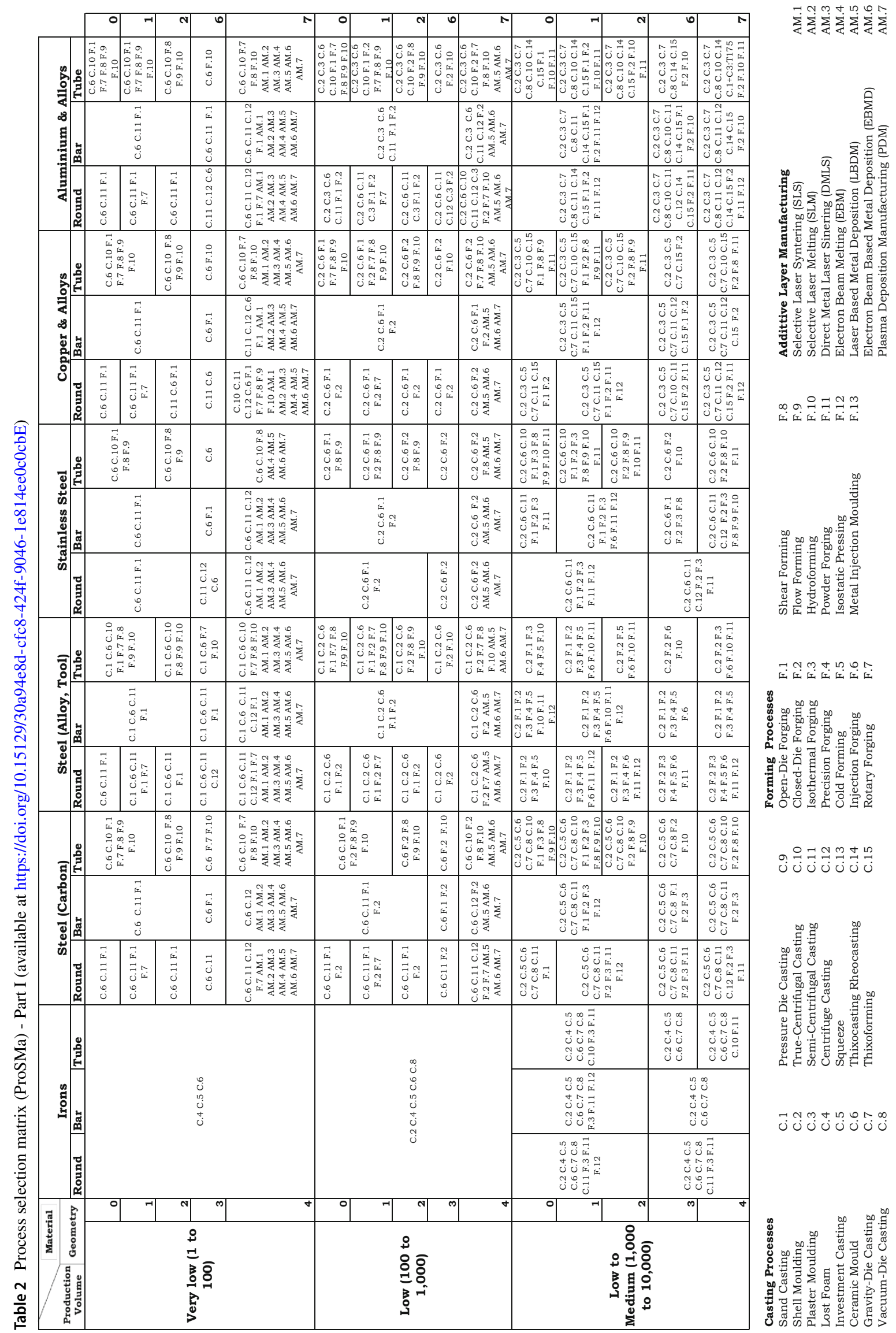









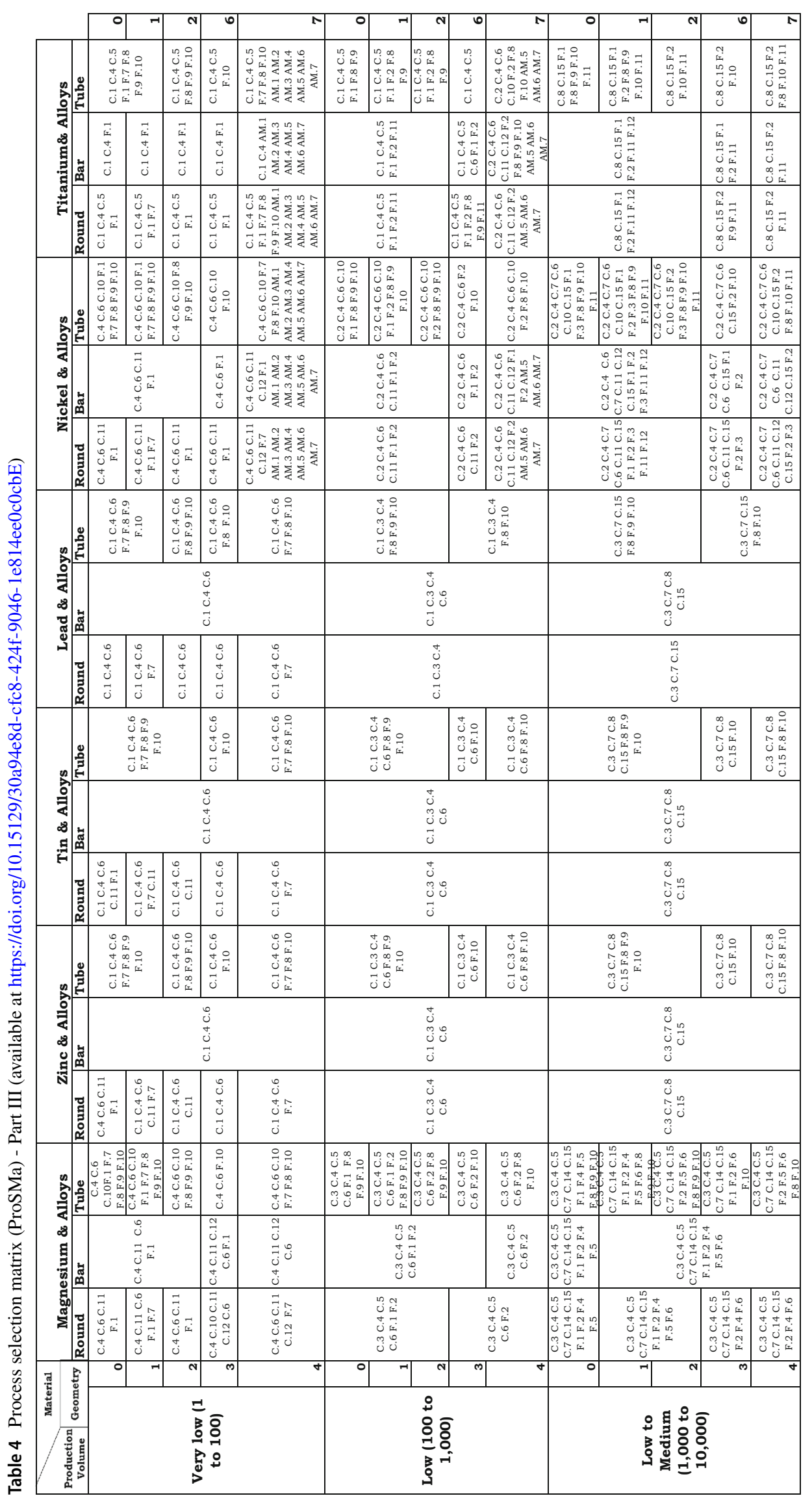

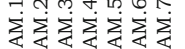

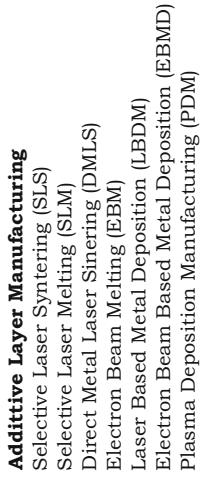

$\infty, a ㅋ ㅜ ㄱ$

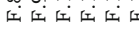

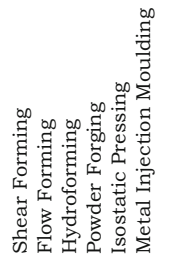

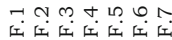

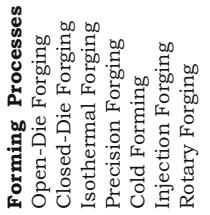

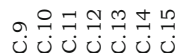

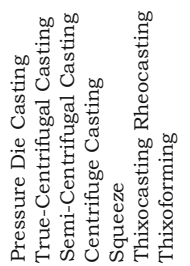

تَن

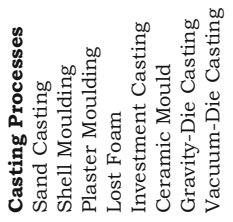







Table 6 Linguistic evaluation scale used in fuzzy logic

\begin{tabular}{ll}
\hline Linguistic evaluation & Value \\
\hline High & 5 \\
Moderate to high & 4 \\
Moderate & 3 \\
Low to moderate & 2 \\
Low & 1 \\
\hline
\end{tabular}

probabilistic behaviour: the medium levels (2 and 3) are associated with the normal process capabilities, so the assigned probability to be achieved is 1 . The extreme ranges (i.e. 1 and 4) are the maximum and minimum capabilities reachable by the process. Between these values and the normal operative ranges (i.e. between 1 and 2 and between 3 and 4), the fuzzy probability needs to be taken into consideration, by assuming a linear behaviour between the two points. Using the Eqs. 1, 2, 3, 4, 5 and 6, it is possible to determine the process compatibility by assessing the required levels, for a number of product attributes, and comparing them with four capabilities levels (fuzzy trapezoidal shape) of the processes. The following four characteristics are taken into consideration:

- Technological attributes (tolerances and surface roughness)

- Feasibility attributes (minimum section and weight)

- Resulting mechanical properties

- Process costs (tooling, equipment and labour)

The first two categories are numerical, meanwhile the last two are usually evaluated on a qualitative scale. The linguistic evaluation scale is showed in Table 6: in this way it is possible to translate qualitative evaluation into a numerical one and using the result for probability calculation. The calculated compatibility for each characteristic are combined to a single compatibility value using Eqs. 5 and 6. The compatibility values are ranked using a weighing scale shown in Table 7. As mentioned previously, [30] introduced a method of combining measures of possibility and necessity presented here as Eq. 7.

$C_{i}=P_{i}(\beta) N_{i}(1-\beta)$

Table 7 Weighing scale used in fuzzy logic

\begin{tabular}{ll}
\hline Features Importance category & Weight \\
\hline Very important & 5 \\
Important & 4 \\
Medium important & 3 \\
Low important & 2 \\
Almost negligible & 1 \\
\hline
\end{tabular}

Table 8 Possibility probability calculation for Request $=$ Value

\begin{tabular}{ll}
\hline Request $=$ Valuey & Possibility \\
\hline If Level $1 \leq$ Request $<$ Level 2 & $\left(\right.$ Req - Lev $\left._{1}\right) /\left(\right.$ Lev $_{2}-$ Lev $\left._{1}\right)$ \\
If Level $2 \leq$ Request $\leq$ Level 3 & 1 \\
If Level $3<$ Request $\leq$ Level 4 & $1-\left(\right.$ Req $^{\prime}-$ Lev $\left._{3}\right) /\left(\right.$ Lev $_{4}-$ Lev $\left._{3}\right)$ \\
If Request $<$ Level 1 & 0 \\
If Request $>$ Level 4 & 0 \\
\hline
\end{tabular}

For the each of the $i$ th attribute: $C_{i}$ is the compatibility for the single attribute; $P_{i}$ is the possibility probabilistic evaluation of the request; $N_{i}$ is the necessity probabilistic evaluation; $\beta$ is the 'optimism' level.

Eqs. 1, 2, 3 and 4 , that calculate the single feature probability, need to be modified depending on the request form. If the request is a single value $\left(R_{e q}\right)$, the possibility and necessity values are calculated as in Tables 8 and 9 , using the four capabilities levels $\left(L e v_{1}, L e v_{2}, L e v_{3}, L e v_{4}\right)$. Similarly, if the request is smaller or bigger than certain values, the possibility and necessity formulas need to be modified accordingly, as displayed in Tables 10, 11, 12 and 13 respectively.

\section{Case study: application of ProGeMa3 methodology}

The ProGeMa3 methodology has been applied to the manufacturing of a control valve (Fig. 5), which was a product of a collaborating company. Commercial confidentiality prevents some details of the components and its production target being reported here.

The following paragraphs describe the application of each step of the ProGeMa3 method.

Step 1: component screening-Investigating the current production parameters (general details given in Table 14), as a consequence of which valve cage (Fig. 6) was found to have highest machining rate and raw material cost impact ratio among control valve's components (Fig. 5) production.

The control valve's cage has a high number of variants in size (from 50 to $600 \mathrm{~mm}$ ) and material (various Stainless Steel), so the most frequently produced size and material

Table 9 Necessity probability calculation for Request $=$ Value

\begin{tabular}{ll}
\hline Request $=$ Value & Necessity \\
\hline If Level $1 \leq$ Request $<$ Level 2 & $1-\left(\right.$ Req $^{\prime}$ Lev $\left._{1}\right) /\left(\right.$ Lev $_{2}-$ Lev $\left._{1}\right)$ \\
If Level $2 \leq$ Request $\leq$ Level 3 & 1 \\
If Level $3<$ Request $\leq$ Level 4 & $1-\left(\right.$ Req - Lev $\left._{3}\right) /\left(\right.$ Lev $_{4}-$ Lev $\left.\left._{3}\right)\right)$ \\
If Request $<$ Level 1 & 0 \\
If Request $>$ Level 4 & 0 \\
\hline
\end{tabular}


Table 10 Possibility probability calculation for Request $<$ Value

\begin{tabular}{ll}
\hline Request $<$ Value & Possibility \\
\hline If Level $1 \leq$ Request $<$ Level 2 & $\left(\right.$ Req $_{-}-$Lev $\left._{1}\right) /\left(\right.$ Lev $_{2}-$ Lev $\left._{1}\right)$ \\
If Level $2 \leq$ Request $\leq$ Level 3 & 1 \\
If Level $3<$ Request $\leq$ Level 4 & 1 \\
If Request $<$ Level 1 & 0 \\
If Request $>$ Level 4 & 1 \\
\hline
\end{tabular}

combination has been selected $(300 \mathrm{~mm}$ and 420 stainless steel) for the case study. The production volume is less than 100 units per year. The extensive machining and the very high material cost (i.e. stainless steel) are the main reasons for the selection of this component. Currently, the component is forged as solid blank and machined (turning and drilling) to the final shape.

Step 2: material selection-In the valve cage case, the material selection has been constrained by three factors

- Application environment: material is selected by the required erosion/corrosion resistance, particularly for fracking pumps, centrifugal and vertical pumps. Changing material requires extensive testing.

- Production variants: all the components already include different material variants, selected by specific customer requests.

- Industrial sector: material choice is dictated by the customer request for pumps/vales (i.e. Oil \& Gas sector) and product standards for valves (i.e. Nuclear sector). Customer are unwilling to accept any new material for these components, without extensive validation.

Therefore, the material selection remains unchanged (i.e. Stainless Steel). Step 3: process selection matrix application-As showed in Table 14, the selected component can be classified using the Table 1 as well as the defined material categories and production volume ranges in. Valve cage can be classified as:

- Geometry: T1. Tubular with a single change of section at the end (Table 1)

- Material: stainless steel

- Production volume: low ( $<100$ units per year).

Table 11 Necessity probability calculation for Request $<$ Value

\begin{tabular}{ll}
\hline Request $<$ Value & Necessity \\
\hline If Level $1 \leq$ Request $<$ Level 2 & $1-\left(\right.$ Req - Lev $\left._{1}\right) /\left(\right.$ Lev $_{2}-$ Lev $\left._{1}\right)$ \\
If Level $2 \leq$ Request $\leq$ Level 3 & 0 \\
If Level $3<$ Request $\leq$ Level 4 & 0 \\
If Request $<$ Level 1 & 1 \\
If Request $>$ Level 4 & 0
\end{tabular}

Table 12 Possibility probability calculation for Request $>$ Value

\begin{tabular}{ll}
\hline Request $>$ Value & Possibility \\
\hline If Level $1 \leq$ Request $<$ Level 2 & $1-\left(\right.$ Req - Lev $\left._{3}\right) /\left(\right.$ Lev $_{4}-$ Lev $\left._{3}\right)$ \\
If Level $2 \leq$ Request $\leq$ Level 3 & 1 \\
If Level $3<$ Request $\leq$ Level 4 & 1 \\
If Request $<$ Level 1 & 0 \\
If Request $>$ Level 4 & 1 \\
\hline
\end{tabular}

Using these input, the ProSMa (Tables 2 and 3), identify a cell that contains the following potential NNS process:

- Sand casting

- Lost foam casting

- Investment casting

- Ceramic moulding

- Flow forming

Table 14 shows also the application of ProSMa to the other components of the assembly.

Step 4: process compatibility evaluation-To apply the fuzzy logic screening and ranking of these candidates, the following characteristics (for both component and processes) have been selected.

- Radial (or planar) tolerance $( \pm \mathrm{mm})$ (numerical evaluation).

- Axial (or vertical) tolerance $( \pm \mathrm{mm})$ (numerical evaluation).

- Surface roughness (Ra) (numerical evaluation).

- Section thickness (mm) (numerical evaluation).

- Weight (kg) (numerical evaluation).

- Resulting mechanical proprieties (linguistic evaluation).

- Tooling cost (linguistic evaluation).

- Equipment cost (linguistic evaluation).

- Labour cost (linguistic evaluation).

These characteristics will evaluated for the requested characteristics (product and targets) and process working ranges (fuzzy sets) and compared between them. The four levels required for defining the centrifugal casting's fuzzy sets have been drawn from the literature. In particular, for tolerances and roughness, the fuzzy ranges have been

Table 13 Necessity probability calculation for Request $>$ Value

\begin{tabular}{ll}
\hline Request $>$ Value & Necessity \\
\hline If Level $1 \leq$ Request $<$ Level 2 & 1 \\
If Level $2 \leq$ Request $\leq$ Level 3 & $($ Req - Lev 3$) /($ Lev $4-$ Lev 3$)$ \\
If Level $3<$ Request $\leq$ Level 4 & 1 \\
If Request $<$ Level 1 & 1 \\
If Request $>$ Level 4 & 0 \\
\hline
\end{tabular}


Fig. 5 Control valve (case study): assembly and components' nomenclature

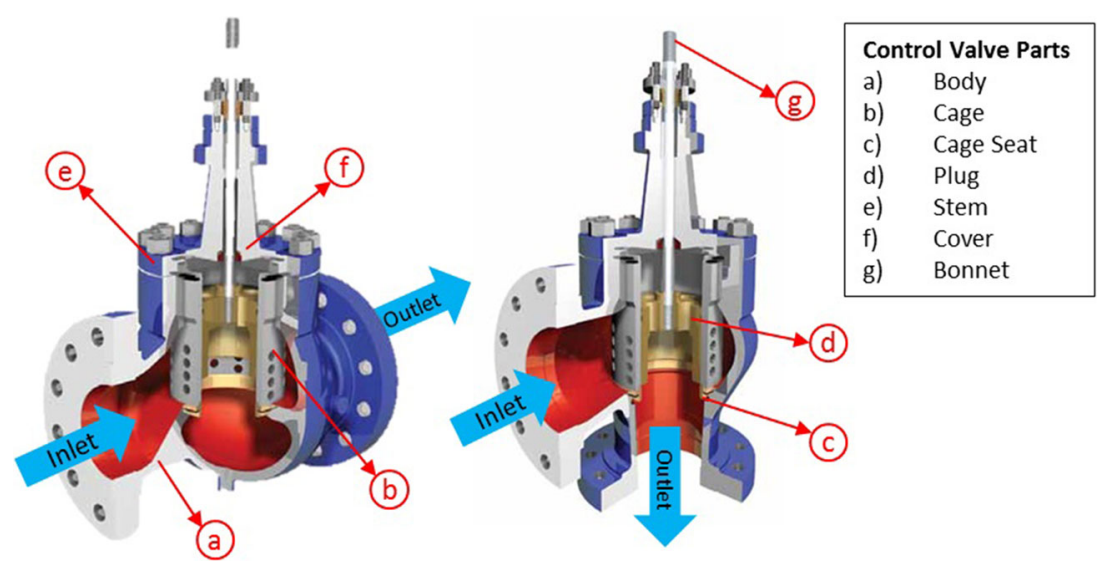

derived from $[12,18,39,61,62,70,71]$; meanwhile workable weights and section thickness ranges have been defined for the investigated processes as in $[4,12,14,39,61$, $62,67,68,70]$.The resulting mechanical properties $[2,4,39$, $57,61,76]$ as well as the tooling, equipment and labour cost $[4,15,39,61,70]$ have been evaluated to define qualitative levels (i.e. low, moderate to low, moderate, moderate to high, high).

The requested levels of the considered characteristics, are showed in Table 15. The component requirements are defined by industrial data (tolerances, roughness, dimensions and weight), quality (mechanical properties) and production (costs) targets. Required tolerances, surface roughness, workable thickness and workable weight levels have been taken from current component properties for the selected size (300 $\mathrm{mm}$ in diameter). Resulting mechanical proprieties have been set to be "bigger than" the estimation of the current and required mechanical proprieties. Similarly, Costs required levels have been set to be "lower than" the current manufacturing costs.

Table 15 also displays the weighting coefficients for the considered features. The highest weight (value = 5) has been given to the workable weight and section thickness, because these properties determine the viability of components manufactured with a given process. The tolerances and surface roughness have been assigned with a high relevance (value $=4$ ), because an NNS approach should aim to achieve properties as close as possible to the request specification. Medium (value $=3$ ) has been given to the mechanical properties, because of the low resistance required by the component. The compatibility weights for the labour, equipment and tooling costs have been set to medium (value $=3$ ) because of the low level of approximation. The $\beta$ coefficient (7) has been set to a constant with a value of 0.5 based on the recommendation of [30].

The target requirements and relative weighting coefficients have been defined in collaboration with the case study company.

Compatibilities for single features $(1,2,3,4)$ and process (5 and 6) could be calculated using the developed fuzzy sets and requirements data (Table 15).

For each process, the total compatibility (7) is calculated using the selected weights (Table 15) and calculating the ranked weight (6).

\section{Results and discussion}

Table 16 displays the total compatibility values of the case study component with each NNS candidate process identified and ranked by the ProGeMa3.

Table 14 ProSMa application to control valve's component manufacturing

\begin{tabular}{lllll}
\hline Component & Production volume & Material & Geometry & Feasible processes (ProSMa) \\
\hline Bonnet & $10-100$ & Stainless steel & T7 & C.6 C.10 F.7 F.8 F.10 AM.1 AM.2 AM.3 AM.4 AM.5 AM.6 AM.7 \\
Body & $10-100$ & Alloy steel & T6 & C.6 F.7 F.10 \\
Disc & $10-100$ & Stainless steel & T7 & C.6 C.10 F.7 F.8 F.10 AM.1 AM.2 AM.3 AM.4 AM.5 AM.6 AM.7 \\
Seat & $10-100$ & Stainless steel & T1 & C.6 C.10 F.1 F.7 F.8 F.9 F.10 \\
Plug stem & $10-100$ & Stainless steel & R2 & C.6 C.11 F.1 \\
Cage (Gasket) & $10-100$ & Stainless steel & T1 & C.1 C.4 C.5 C.6 C.11 F.9 \\
\hline
\end{tabular}




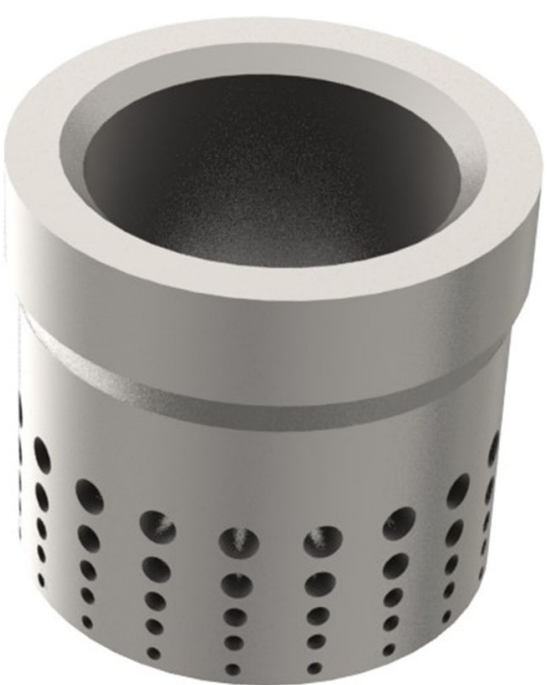

Fig. 6 Component selection for NNS investigation (Cage)

The zero results for sand casting, investment casting and lost foam casting suggest they are not appropriate for the NNS manufacturing of valve cage.

This because tolerances, roughness and mechanical properties compatibly values make these casting process less suitable for this application. For sand casting and lost foam casting, the tolerances, roughness and mechanical properties capabilities are not sufficient for allowing the process to produce the requested characteristics. Investment casting is also not appropriate because of its high costs.

However, three processes (centrifugal casting, ceramic moulding and flow forming) are considered compatible with the requirements of the valve cage manufacturing. The centrifugal casting process is the most suitable (0.92), followed by flow forming (0.77) and ceramic moulding (0.68) (Fig. 7).

Figure 8 shows the compatibility of the single features for all the considerer processes. Centrifugal casting satisfies

Table 15 Required levels for process compatibly evaluation through fuzzy logic

\begin{tabular}{lll}
\hline Component request and weights & Requests & $\begin{array}{l}\text { Weighting } \\
\text { factors }\end{array}$ \\
\hline Radial tolerance $( \pm \mathrm{mm})$ & 0.25 & 4 \\
Axial tolerance $( \pm \mathrm{mm})$ & 0.25 & 4 \\
Surface roughness $(\mathrm{Ra})$ & 1.6 & 4 \\
Workable section thickness $(\mathrm{mm})$ & 80 & 5 \\
Workable weight $(\mathrm{kg})$ & 360 & 5 \\
Resulting mechanical proprieties & $\geq 4$ & 3 \\
Tooling cost & $\leq 3$ & 3 \\
Equipment cost & $\leq 3$ & 3 \\
Labour cost & $\leq 3$ & 3 \\
\hline
\end{tabular}

Table 16 Compatibility rankings of the processes by fuzzy logic

\begin{tabular}{ll}
\hline Manufacturing process & Total compatibility (case study II) \\
\hline Centrifugal casting & 0.92 \\
Ceramic moulding & 0.68 \\
Flow forming & 0.77 \\
Sand casting & 0.00 \\
Lost foam casting & 0.00 \\
Investment casting & 0.00 \\
\hline
\end{tabular}

almost completely the requested levels. Flow forming exceed centrifugal casting capabilities on some of the features. However, similarly to ceramic moulding, different ranges in workable thickness and weight reduce their total compatibility (given the weights applied to the coefficients). This results in the highest compatibility being for centrifugal casting in comparison with ceramic moulding or flow forming process.

As a direct result of this analysis, centrifugal casting was adopted for valve cage production generating $26.5 \%$, saving 490 machining hours and 18.9 tons of raw material. The NNS process feasibility study and application details can be found in [46].

In comparison with the literature, ProGeMa3 investigating only metals (similarly to [25] and [12]) although they are both restricted in scope to casting processes. Swift and Booker [70] investigate metals, composites and plastic, but the approach is restricted to traditional manufacturing processes. The methodologies of [1, 30] and [5] can be applied to all material and target process.

The general methodologies ([1,30] and [5]) are also the most adaptable ones, and the casting-dedicated approaches the least flexible. Giachetti [30] uses fuzzy logic approach to process selection, ranking the process candidates feasibility for the target production, meanwhile [70] use a combination of a selection matrix (used as a filter) and cost analysis (use a final decisional criteria). In contrast, ProGeMa3 combine their key features, using a combination of process selection matrix and fuzzy logic, to rank the process candidates, using NNS criteria.

The impact of a product requirements' precision on the process selection is lower on the less quantitative (based on archival data) or qualitative methodologies. Meanwhile it heavily influences the quantitative ones, for example in [5] by selecting the criteria and targets of the value functions. The procedures based on fuzzy logic are highly influenced by the dimensional precision demanded by the product requirements and consequently has a significant impact on the the ranking process.

Differently from [12, 70] and [1], ProGeMa3 does not rely only static archival data, which are less suitable for 
Fig. 7 Ranking of process compatibilities for the NNS manufacturing of the case study component

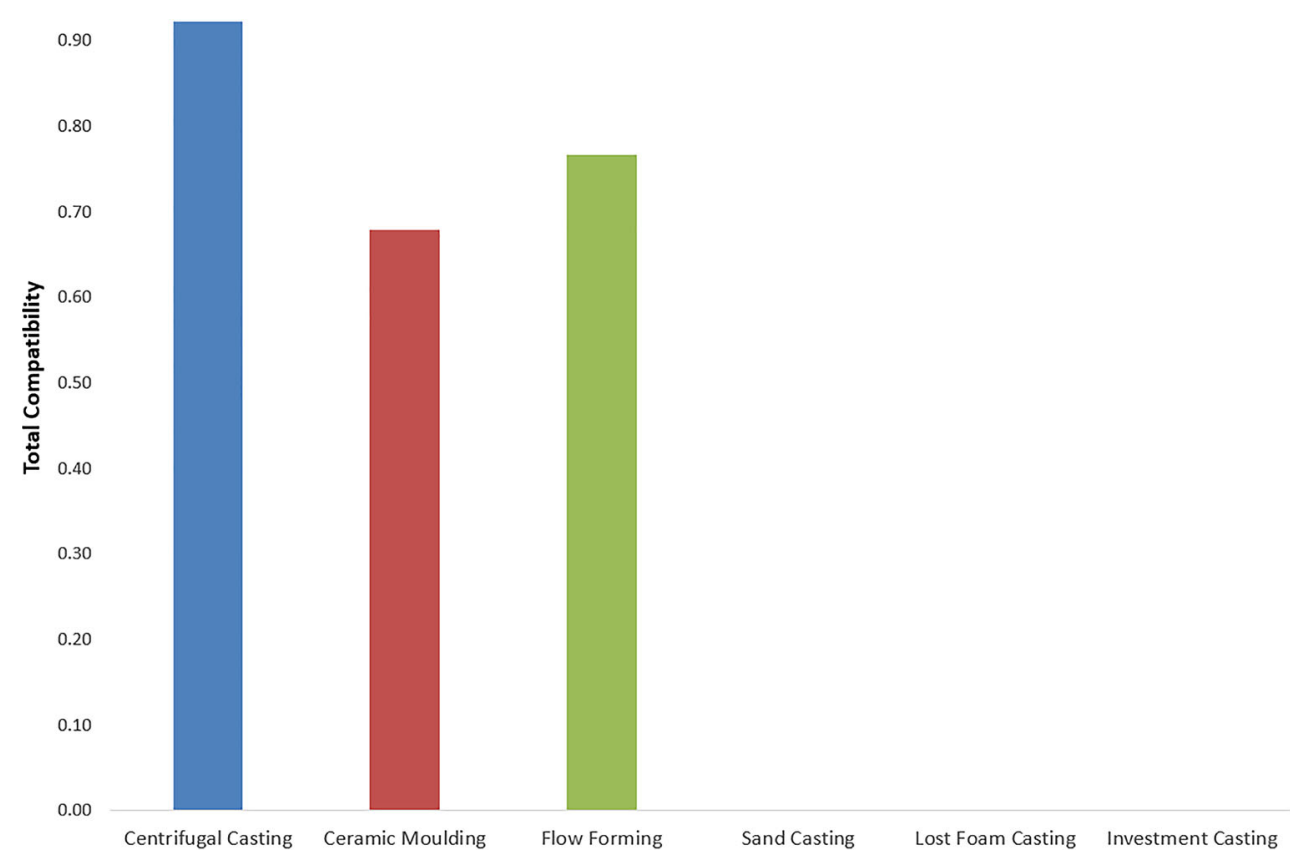

defining new and emerging processes. Fuzzy (for example, [30] and ProGeMa3) and analytical (for example [5]) approaches can adapt to experimental data and be updated in an agile manner.

ProGeMa3 selects the material before process selection (like [12, 70] and [25]), while, [30] and [5] approaches have interactive process and material selections.

Albiñana and C. Vila [1], and Er and Dias [25] use both qualitative methods, the first using a knowledge-based framework selection, the second a rule-based selection. The different methodologies consider different attributes for selecting the process. ProGeMa3 use cost, product geometry, mechanical properties, production volume and materials (used into the selection matrix phase. Giachetti [30] take into considerations more variables, material, product geometry, process features, mechanical properties, production volume and cost into its fuzzy logic model.
Fig. 8 Process compatibilities breakdown for the NNS manufacturing of the case study component
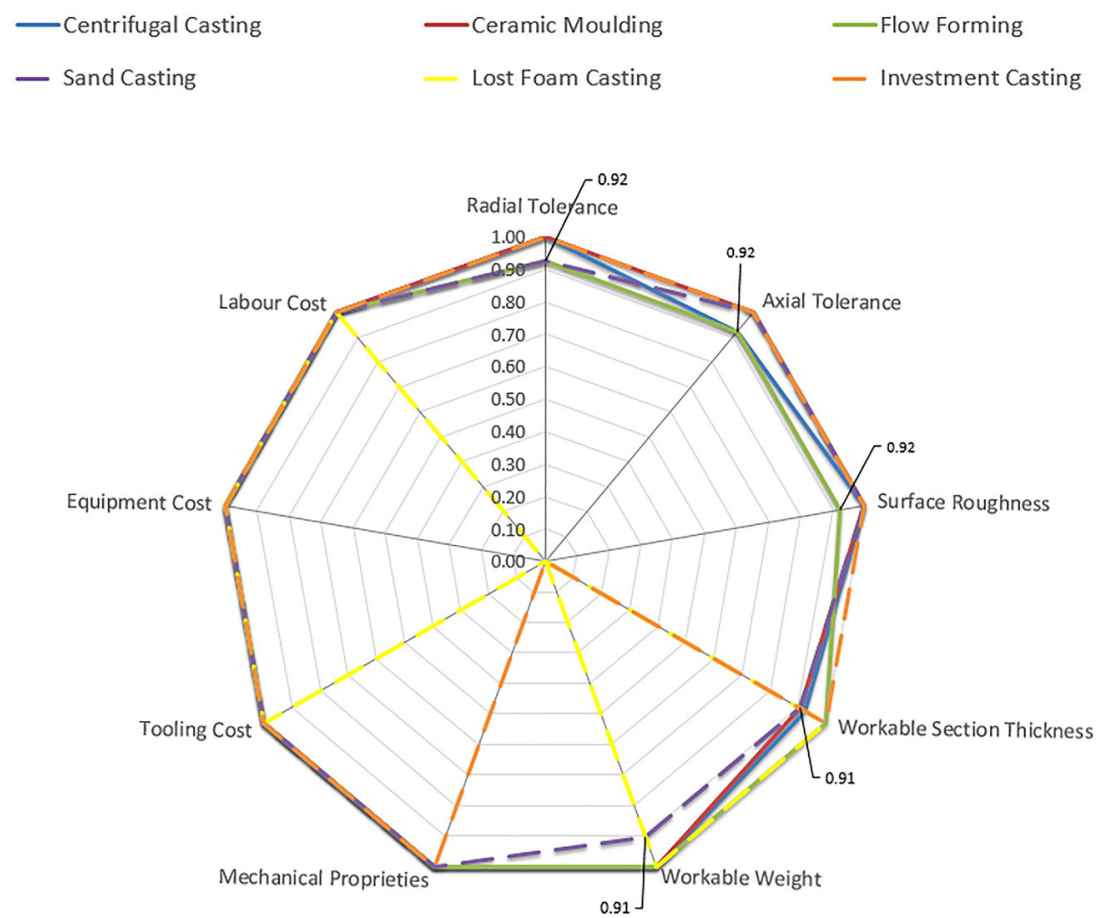
Different from [30] and [5], ProGeMa3 is not be able to assess the optimal selection, as it restricts its working field through ProSMa.

\section{Conclusion}

The ProGeMa3 methodology has been successfully applied to an industrial case study. The assumption of material constancy during the process selection is viable in a case study application and align with previously reported approaches in the literature.

The methodology can be potentially expanded to include ceramic and plastic materials, including their dedicated processes. Similarly, sheet manufacturing and continuous processes could be added into the process selection matrix.

The methodology has potential to be automated as an online service with a graphical user interface to facilitate its usage by non-expert users.

Customer needs and market influence are difficult to quantify, although they have a great impact on requirement definition and supply chain stability. Complexity of quantifying this kind of information makes it difficult to include these characteristics in a quantitative methodology. Potential quality enhancement (related to the application of different processes) is difficult to quantify during the selection stage. Similarly, production volume variabilities and changes in relationship to any other modifications.

Similarly, environmental and energy impacts are difficult to quantify at process selection stage, although they have a great impact on the supply chain sustainability. In this sense, ProGeMa3 can be coupled with other quantitative methodologies, such as life-cycle assessment (LCA) to quantify the process selection influence on environmental impact. However, the dependency of supply chain management and product life-cycle on product requirements, suppliers and external factors (such as governmental policy and market regulations) make these factors strongly "case-dependent" and difficult to categorize.

The combination of selection matrix and fuzzy logic provides a very efficient mechanism for quickly focusing the process selection on a small number of potential candidates. Once the process databases have been created, the ProGeMa3 methodology reduces the amount of subjectivity in the process and consequently supports non expert-users [60]. Requirements definition results critical as they could lead to different results in the process selection. In this sense, availability of information is critical for the application of section methodologies.

The ProSMa matrix is provided for download with source files so other researches can expand and update the methodology (electronically available at [45]).
Acknowledgments This research is a part of Research Chair in Advanced Forming and Forging project (EPSRC funding: EP/H03465X/1), conducted by the University of Strathclyde (DMEM) and several partners.

Funding information The authors want to thank WARC (Weir Advanced Research Centre) and Weir Group PLC of Glasgow for the fundamental and continuous support in this research.

Open Access This article is distributed under the terms of the Creative Commons Attribution 4.0 International License (http:// creativecommons.org/licenses/by/4.0/), which permits unrestricted use, distribution, and reproduction in any medium, provided you give appropriate credit to the original author(s) and the source, provide a link to the Creative Commons license, and indicate if changes were made.

\section{References}

1. Albiñana JC, Vila C (2012) A framework for concurrent material and process selection during conceptual product design stages. Mater Des 41:433-446

2. Allen AJ, Swift KG (1990) Manufacturing process selection and costing. Proc Institut Mech Eng Part B: J Eng Manuf 204(143): 143-148

3. Altan T, Allen Miller R (1990) Design for forming and other near net shape manufacturing processes. CIRP Ann Manuf Technol 39(2):609-620

4. Altan T (2005) Cold and hot forging. ASM International

5. Ashby MF (2000) Multi-objective optimization in material design and selection. Acta Mater 48(1):359-369

6. Cebon D, Ashby MF (1993) Materials selection in mechanical design. Le J de Physique IV 3(C7):C7-1-C7-9

7. Balendra R (1993) Injection-chamber to die-cavity interface for injection forming. Int $\mathrm{J}$ Mach Tools Manuf 33(6):753760

8. Balendra R, Yi Q (2004) Injection forging: engineering and research. J Mater Process Technol 145(2):189-206

9. Bensmaine A, Dahane M, Benyoucef L (2013) A simulationbased genetic algorithm approach for process plans selection in uncertain reconfigurable environment 46(9):19611966

10. Joran W (2016) Booth: Idetc2016-60407, the design for additive manufacturing worksheet. In: Proceedings of the ASME 2016 international design engineering technical conferences \& computers and information in engineering conference IDETC/CIE 2016 (August)

11. Brechet Y, Bassetti D, Landru D, Salvo L (2001) Challenges in materials and process selection. Prog Mater Sci 46(3-4):407428

12. Campbell J (2000) The concept of net shape for castings. Mater Des 21(4):373-380

13. Chakraborty S, Dey S (2007) QFD-based expert system for non-traditional machining processes selection. Expert Syst Appl 32(4):1208-1217

14. Chang T-C, Wysk RA, Wang H-P (1998) Computer-aided manufacturing. Prentice Hall, pp 596 -598

15. Cominotti R, Gentil E (2008) Near net shape technology: an innovative opportunity for the automotive industry. Robot Comput Integr Manuf 24(6):722-727

16. Cooper WW, Sinha KK, Sullivan RS (1992) Measuring manufacturing: indexes in high-technology for evaluation. Interfaces 22(4):38-48 
17. Darwish SM, El-Tamimi AM (1996) The selection of the casting process using an expert system. Comput Ind 30(30):77-86

18. Davidson MJ, Balasubramanian K, Tagore GRN (2008) Experimental investigation on flow-forming of AA6061 alloy-a Taguchi approach. J Mater Process Technol 200(1-3):283-287

19. Daws KM, Al-dawood ZI, Al-kabi SH (2008) Selection of metal casting processes: a fuzzy approach. Jordan J Mech Indust Eng 2(1):45-52

20. Doege E, Thalemann J (1989) Near net-shape forming in sheetmetal forming and forging. CIRP Ann Manuf Technol 38(2):609616

21. Douglas R, Kuhlmann D (2000) Guidelines for precision hot forging with applications. J Mater Process Technol 98(2):182-188

22. Dubois D, Prade H (2012) Possibility theory. Springer

23. Edwards P, O'Conner A, Ramulu M (2013) Electron beam additive manufacturing of titanium components: properties and performance. J Manuf Sci Eng 135(6):061016

24. ElMaraghy WH, Urbanic RJ (2003) Modelling of manufacturing systems complexity. CIRP Ann Manuf Technol 52(1):363-366

25. Er A, Dias R (2000) Rule-based expert system approach to process selection for cast components. Knowl-Based Syst 13(4):225-234

26. Esawi AMK, Ashby MF (1998) Computer-based selection of manufacturing processes: methods, software and case studies. Proc Institut Mech Eng Part B: J Eng Manuf 212(8):595-610

27. Esawi AMK, Ashby MF (2003) Cost estimates to guide preselection of processes. Mater Des 24(8):605-616

28. Facchini L, Magalini E, Robotti P, Molinari A (2009) Microstructure and mechanical properties of Ti-6Al-4V produced by electron beam melting of pre-alloyed powders. Rapid Prototyp $\mathrm{J}$ 15(3):171-178

29. Ford S, Despeisse M (2016) Additive manufacturing and sustainability: an exploratory study of the advantages and challenges. J Clean Prod 137:1573-1587

30. Giachetti R (1998) A decision support system for material and manufacturing process selection. J Intell Manuf 9:265-276

31. Gibson I, Rosen DW, Stucker B (2010) Additive manufacturing technologies

32. Guenov MD (2002) Complexity and cost effectiveness measures for systems design. Manufacturing Complexity Network Conference, $9-10$

33. Gur M, Tirosh J (1982) Plastic flow instability under compressive loading during shear spinning process. J Eng Indus-Trans ASME 104(1):17-22

34. Han X, Hua L (2009) Comparison between cold rotary forging and conventional forging. J Mech Sci Technol 23(10):2668-2678

35. Hayama M, Kudo H (1979) Experimental Study of Tube Spinning. Bull JSME 22(167):769-775

36. Holland P, Standring PM, Long H, Mynors DJ (2002) Feature extraction from STEP (ISO 10303) CAD drawing files for metalforming process selection in an integrated design system. $\mathbf{J}$ Mater Process Technol 125-126:446-455

37. Hwang W-s, Stoehr RA (1988) Molten metal flow pattern prediction for complete solidification analysis of near net shape castings. Mater Sci Technol 4(3):240-250

38. Kalpakcioglu S (1961) On the mechanics of shear spinning. Trans ASME J Eng Ind 83:125-130

39. Kalpakjian S, Schimd SR (2009) Manufacturing engineering and technology, 6 edn. Prentice Hall

40. Karthik S, Chung CW, Ramani K, Tomovic MM (2003) Methodology for metalcasting process selection. SAE 2003 Trans J Mater Manuf 112(5):12

41. Kerbrat O, Mognol P, Hascoet JY (2008) Subtractive processes : application to hybrid modular tooling. Solid freeform fabrication symposium Proceedings, 519-530

42. Kutz M (2002) Handbook of materials selection
43. Long H, Mynors DJ, Holland P, Standring PM (2002) Forming feature representation and process selection in cold extrusion. $\mathrm{J}$ Mater Process Technol 125-126:456-463

44. Lovatt AM, Shercliff HR (1998) Manufacturing process selection in engineering design. Part 1: the role of process selection. Mater Des 19(5-6):205-215

45. Marini D (2019) Process selection matrix for near net shape manufacturing. https://doi.org/10.15129/30a94e8d-cfc8-424f-9046-1e8 $14 \mathrm{ee} 0 \mathrm{c} 0 \mathrm{cb}$

46. Marini D, Corney JR (2017) A methodology for near net shape process feasibility assessment. Prod Manuf Res 5(1):390-409

47. Marini D, Cunningham D, Corney JR (2017) Near net shape manufacturing of metal: a review of approaches and their evolutions. In: Proceedings of the institution of mechanical engineers, Part B: journal of engineering manufacture

48. Martínez-Olvera C (2012) An entropy-based approach for assessing a product's BOM blocking effect on a manufacturing process flow. Int J Product Res 50(4):1155-1170

49. Mathur P, Apelian D, Lawley A (1989) Analysis of the spray deposition process. Acta Metallurgica 37(2):429-443

50. Milani AS, Shanian A, Lynam C, Scarinci T (2013) An application of the analytic network process in multiple criteria material selection. Mater Des 44:622-632

51. Music O, Allwood JM, Kawai K (2010) A review of the mechanics of metal spinning. J Mater Process Technol 210(1):3-23

52. Niebling F, Geiger OM (2002) Analyzing the DMLS-process by a macroscopic FE-model. In: Proc. of 13th solid freeform fabrication, pp 384-391

53. Onodera S, Sawai K (1992) Current cold-forging techniques for the manufacture of complex precision near-net-shapes. J Mater Process Technol 35:385-396

54. Perzyk M, Meftah OK (1998) Selection of manufacturing process in mechanical design. J Mater Process Technol 76(1997):198-202

55. Pham DT, Gault RS (1998) A comparison of rapid prototyping technologies. Int J Mach Tools Manuf 38(10-11):1257-1287

56. Plancak ME, Vilotic DZ, Stefanovic MC, Movrin DZ, Kacmarcik IZ (2012) Orbital forging - a plausible alternative for bulk metal forming. J Trends Develop Mach Assoc Technol 16(1):35-38

57. Podder B, Mondal C, Ramesh Kumar K, Yadav DR (2012) Effect of preform heat treatment on the flow formability and mechanical properties of AISI4340 steel. Mater Des 37:174-181

58. Rao RV, Padmanabhan KK (2007) Rapid prototyping process selection using graph theory and matrix approach. J Mater Process Technol 193(1-3):81-88

59. Ravi B (2005) Metal casting: computer-aided design and analysis. PHI Learning Pvt. Ltd.

60. Sáenz DC, Castillo DC, Romeva CR, Macià JL (2015) A fuzzy approach for the selection of non-traditional sheet metal cutting processes. Expert Syst Appl 42(15-16):6147-6154

61. Schey JA (1999) Introduction to manufacturing processes. McGraw-Hill

62. Schuler (1998) Metal forming handbook, vol 3

63. Sheljaskov S (1994) Current level of development of warm forging technology. J Mater Process Technol 46(1-2):3-18

64. Shercliff HR, Lovatt AM (2001) Selection of manufacturing processes in design and the role of process modelling. Prog Mater Sci 46(3-4):429-459

65. Sirilertworakul N, Webster PD, Dean TA (1993) A knowledge base for alloy and process selection for casting. Int J Mach Tools Manuf 33(3):401-416

66. Sivanandini M, Dhami SS, Pabla BS (2012) Flow forming of tubes-a review. Int J 3(5):1-11

67. Srinivasulu M, Komaraiah M, Rao CSKP (2012) Experimental studies on the characteristics of AA6082 flow formed tubes. J Mech Eng Res 4:192-198 
68. Srinivasulu M, Komaraiah M, Rao CSKP (2012) Experimental investigations to predict mean diameter of AA6082 tube in flow forming process-a DOE approach. IOSR J Eng(IOSRJEN) 2(6):52-60

69. Standring PM (2001) Characteristics of rotary forging as an advanced manufacturing tool. Proc Institut Mech Eng Part B: J Eng Manuf 215(7):935-945

70. Swift KG, Booker JD (2013) Manufacturing process selection handbook. Butterworth-Heinemann

71. Takemasu T, Vazquez V, Painter B, Altan T (1996) Investigation of metal flow and preform optimization in flashless forging of a connecting rod. J Mater Process Technol 59(1-2):95-105

72. Tateno M (1984) Development of large size high quality steels and their future prospect as "near net shape" material. Trans ISIJI 25:97-108

73. Tsinopoulos C, McCarthy I (2006) New product development as a complex system of decisions. J Prod Innov Manag 23(2006):437456

74. Vinodh S, Anesh Ramiya R, Gautham SG (2011) Application of fuzzy analytic network process for supplier selection in a manufacturing organisation. Expert Syst Appl 38(1):272-280
75. Wiendahl HP, Scholtissek P (1994) Management and control of complexity in manufacturing. CIRP Ann Manuf Technol 43(2):533-540

76. Wong C, Dean T, Lin J (2003) A review of spinning, shear forming and flow forming processes. Int $\mathrm{J}$ Mach Tools Manuf 43(14):1419-1435

77. Xu F, Wong YS, Loh HT (2001) Toward generic models for comparative evaluation and process selection in rapid prototyping and manufacturing. J Manuf Syst 19(5):283-296

78. Xu Y, Zhang SH, Li P, Yang K, Shan DB, Lu Y (2001) 3D rigid-plastic FEM numerical simulation on tube spinning. J Mater Process Technol 113(1-3):710-713

79. Yu J-C, Krizan S, Ishii K (1993) Computer-aided design for manufacturing process selection. J Intell Manuf 4(3):199-208

80. Zhang H, Xu J, Wang G (2002) Fundamental study on plasma deposition manufacturing. Surf Coat Technol 171(1-3):112-118

Publisher's note Springer Nature remains neutral with regard to jurisdictional claims in published maps and institutional affiliations. 